\title{
Methodology for the Study of Residual Temperature Stresses in the Butt Contour of a Welded Joint Made of Carbon and High-Alloy Structural Steels during Multi-Pass Welding
}

\author{
Lubov Mironova ${ }^{1, a^{*}}$, Ruslan Nigay ${ }^{2, b}$ and Elena Yakusheva ${ }^{3, c}$ \\ ${ }^{1}$ Moscow Aviation Institute (State National Research University), 4, Volokolamskoe sh., \\ Moscow, 125993, Russia \\ ${ }^{2}$ Russian University of Transport (MIIT), 9, Obraztsova st, Moscow, 127994, Russia \\ ${ }^{3}$ National Research Nuclear University MEPhl, 31, Kashirskoe sh., Moscow, 115409, Russia \\ amironova_lub@mail.ru, bruslan1010@mail.ru, celena.yakusheva77@gmail.com
}

\begin{abstract}
Keywords: Temperature Field, Temperature Stresses, Welding, Welded Joint, Thermal Conductivity Equation, Residual Stresses
\end{abstract}

\begin{abstract}
The method of investigation of residual temperature stresses in the butt contour of the welded joint "shell - plate" made of carbon and high-alloy structural steels during multi-pass welding is described. The temperature problem was solved on the basis of the obtained experimental data of temperature measurement at the reference points of the structure during the application of the rollers. A solution is proposed that takes into account several stages, each of which corresponds to a specific intermediate temperature field due to the peculiarities of welding technology. The evaluation of residual stresses was carried out on the basis of the energy theory of plasticity, taking into account the dependences of the yield strength and elastic modulus of welded metals on temperature.
\end{abstract}

\section{Introduction}

In engineering practice, when designing welded structures made of low-carbon or alloy steel, it is assumed that local deformations themselves cannot exhaust the plasticity reserve of mild steel and, consequently, cracks cannot form [1]. In fact, a sharp drop in thickness in the places of the welded joint of individual parts of the bearing body leads to the formation of local zones with high values of temperature stresses and deformations. The different thickness of the shell structure leads to an uneven distribution of the temperature gradient between the front and inner surfaces, one of which experiences tensile stresses, the other, on the contrary, compressive. In addition, the mechanisms of the occurrence of shrinkage stresses have not been sufficiently studied, which, together with the acting force factors during operation, can be "dangerous" and contribute to the formation of microcracks and further crack development [2].

In many cases, when welding, studies of thermal processes with sufficient accuracy are carried out using the results obtained in the theory of thermal processes [3, 4].

However, exact quantitative dependences cannot be obtained only theoretically, since the assessment of welding stresses and deformations includes consideration of issues of a number of scientific disciplines: thermal conductivity, elasticity, plasticity and creep of metals in a wide range of rapidly changing temperatures [5].

In this paper, methods for estimating residual stresses and deformations caused by welding processes are proposed based on an experimental study of the thermal process of multi-pass welding. 
Characteristics of the investigated welded structure Fig. 1. shows the welded joint and the scheme of applying the weld. A welded joint from the position of mechanics can be conditionally considered as a "shell - plate" joint. A pipe of considerable length is welded to the base of sufficient thickness. The material of the plate is low-carbon steel 20, the material of the shell (tube) is highalloy austenitic steel. The technological properties of these steels in terms of weldability are good.

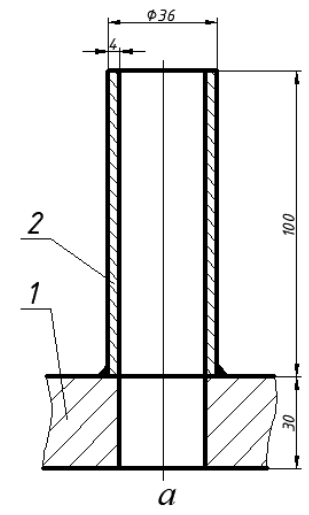

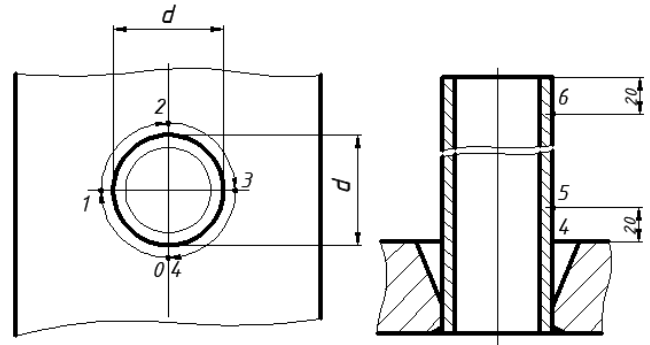

$b$

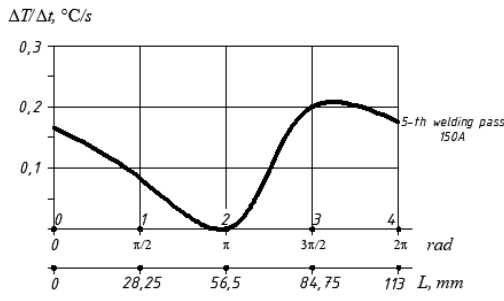

$c$

Fig. 1. Sample (a) and scheme of welding seam (b): 1 -footing; 2 -tube; 3 -distribution of the temperature gradient along the contour of the welded joint "shell - plate"

A distinctive feature of the welded joint is the welding of a pipe of considerable length, which, under conditions of thermal loading by a movable heat source, can lead to significant warping of the structure. The welded joint is non-reversible. The welding method is manual electric arc melting of the electrode. The roller was applied manually with the breakdown of the welded contour into sectors "0-1", "1-2", "2-3", "3-4".

In accordance with the typical welding process, the recommended welding current values range from 20 to 70 A per welding pass. Thus, in one pass of the electrode when welding butt joints, depending on the value of the welding current, the effective thermal power can vary between 22 and $32 \%$.

\section{Purpose and methodology of the experiment}

The purpose of the experiment was to study temperature fields and deformation processes in the zone of technological influence during multi-pass welding of the shell -plate joint under various conditions of thermal loading by welding current: with a decrease and increase in welding current by $6 \%$. within the normal mode.

The experimental work included two stages. The first stage included multi-pass application of the welding seam in accordance with the accepted welding modes.

The second stage consisted in measuring the temperature in the vicinity of the points corresponding to the sector welding in one pass (points 1, 2, 3, 4, Fig. 1 (b)). Temperature measurement was carried out at these points three times with a time interval of 2, 5, 5 minutes after each measurement. The measuring device is a contact digital thermometer of the TK 5-0.1 brand. The measurement accuracy is $1^{\circ} \mathrm{C}$. The welded joint was cooled in natural conditions in air at a temperature of $20^{\circ} \mathrm{C}$.

Table 1 shows the obtained data on the temperature distribution in the welded circuit after the fifth pass. 
Table 1. Temperature change in the contour connection after the fifth pass

\begin{tabular}{|c|c|c|c|c|c|c|c|}
\hline \multicolumn{2}{|c|}{ Time, $\mathrm{s}$} & Temperature, & \multicolumn{5}{c|}{ Characteristic points } \\
\cline { 4 - 8 }$t_{i}$ & $\Delta t$ & ${ }^{\circ} \mathrm{C}$ & 0 & 1 & 2 & 3 & 4 \\
\hline 120 & 120 & $T_{1}$ & 200 & 250 & 245 & 270 & 303 \\
\hline 420 & 300 & $T_{2}$ & 250 & 225 & 245 & 210 & 250 \\
\hline \multicolumn{3}{|c|}{$\Delta T,{ }^{\circ} \mathrm{C}$} & +50 & -25 & 0 & -60 & -53 \\
\hline \multicolumn{3}{|c|}{$\begin{array}{c}\text { Rate of temperature } \\
\text { change }|\Delta T| / \Delta t,{ }^{\circ} \mathrm{C} / \mathrm{sec}\end{array}$} & 0.166 & 0.083 & 0 & 0.2 & 0.176 \\
\hline $\begin{array}{l}\text { Average value of the rate of } \\
\text { temperature change },{ }^{\circ} \mathrm{C} / \mathrm{sec}\end{array}$ & & & & 0.125 & \\
\hline
\end{tabular}

The rate of temperature change was determined by the formula

$$
\frac{\Delta T}{\Delta t}=\frac{\left|T_{2}-T_{1}\right|}{t_{2}-t_{1}} .
$$

Here $T_{1}$ and $T_{2}$ are the temperature at the first and second measurements, $t_{2}$ and $t_{1}$ are the intermediate values of the temperature measurement time: $t_{1}=120 \mathrm{~s}, t_{2}=420 \mathrm{~s}$. The time interval during which the rate of cooling of the welded joint in air was determined was $\Delta t=300 \mathrm{~s}$.

The graph of the temperature gradient distribution along the contour of the welded joint at the fifth pass is shown in Fig. 1 (c). The results of the conducted studies of the distribution of highgradient temperature fields and modeling of the thermal process are presented in [6-8].

For welding processes, in approximate calculations of welding stresses, it is necessary to have dependences of the yield strength and elastic modulus of the metals being welded on temperature. Moreover, residual stresses occur after the complete cooling of the welded structure. The reason for the formation of residual stresses is uneven heating and plastic deformation of the metal during heating [5]. Approaches and methods to the determination of residual stresses in the butt contour of the welded joint will be based on the work $[5,9,10]$

To determine the level of residual stresses in the contour of the weld and the near-weld zone, we will use the methodology described in [10], where the value of the yield strength of the material is taken as the boundary above which residual stresses appear. In accordance with this, we will determine the residual stresses from the equality

$$
\sigma_{\text {res }}=\sigma_{\max }-\sigma_{T}
$$

Where $\sigma_{r e s}$ are residual stresses; $\sigma_{\max }$ are maximum stresses; $\sigma_{T}$ is the yield strength of the material.

The determination of the maximum values of temperature stresses will be determined from the solution of the temperature problem by the graphoanalytic method, using experimental data of temperature measurement at reference points (Fig. 1), taking into account the methodology of work [5]. Thus, the solution of the problem is divided into two stages: the solution of the temperature problem and the problem of stresses in the elastic-plastic formulation.

The graphoanalytical method for determining temperature fields as a function of $T(r, \theta, t)$ in the welded contour of the shell-plate joint is based on a step-by-step solution, each of which corresponds to a specific intermediate value of temperature in time measured experimentally, 
Table 1. Figure $2(a, b, c)$ shows the graphical distribution of temperature fields in the radial direction according to the results of the experiment.

Fig. 2 (a) shows the distribution of the temperature field in the contour of the "shell - plate" welded joint two minutes after applying the roller along the entire contour in accordance with the scheme of Fig. 1. There is a relative uniform temperature redistribution from point " 0 "to point" 4». After 7 minutes after applying the roller over the entire contour, the distribution pattern changed, Fig. 2 (b). The greatest heat capacity is observed in the sector of the contour "2-4".
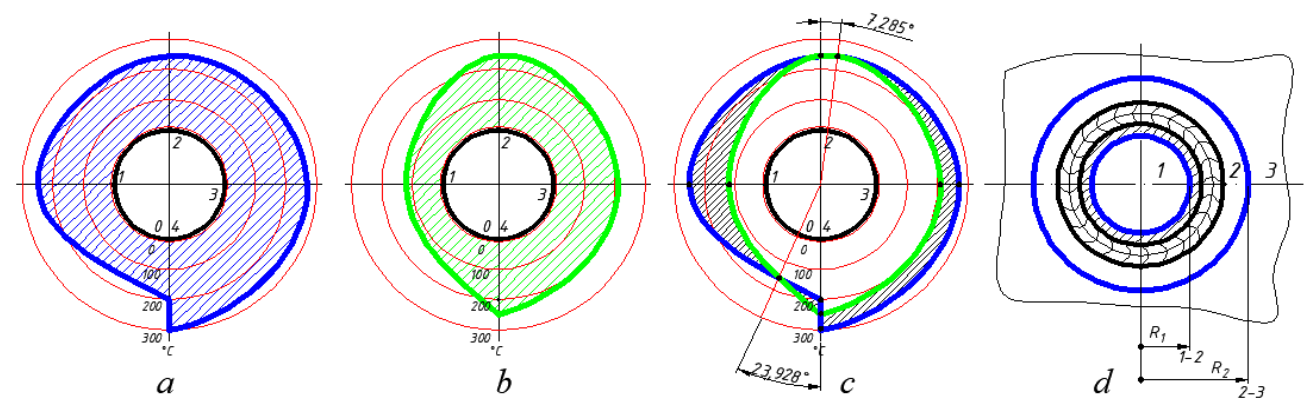

Fig. 2. $a, b, c$-distribution of temperature fields in the radial direction of the contour; $d-$ Stress distribution zones

The distribution of the temperature field in the contour of the welded joint for the time $\Delta t=$ $300 \mathrm{~s}=5 \mathrm{~min}$ is shown in Fig. 2 (c). The highest rate of temperature redistribution is observed in the vicinity of point "1", and in the sector of the contour " $0-1$ " at the intersection of the two curves, the temperature is redistributed to its increase in the coordinate $\varphi=23.928^{\circ} \mathrm{C}$.

In accordance with the theory of welding deformations and stresses [5, 11, 12], it should be assumed that the vicinity of the near-weld zone 0-1-2 is most susceptible to the formation of hot cracks. The stress problem will be considered in the first approximation as axisymmetric, despite the fact that the circular seam is not welded at the same time and the conditions for the formation of metal deformations are different in the zones around the circumference. We divide the section of the welded joint into three zones (Fig. 2 (d)): inner, middle and outer, we have three rings with conditional boundaries of transition from one ring to another in the radial direction 1-2, 2-3.

Zone 2 corresponds to the tensile stress zone in a conventional straight seam and is characterized by high stresses $\sigma_{t 2}$, close to the yield strength. The voltage $\sigma_{r 1-2}$ acts on the inner zone, the voltage $\sigma_{r 2-3}$ acts on the outer zone. Since ring 2 is balanced, then the inequality is valid

$$
\sigma_{r 2-3}>\sigma_{r 1-2} \text {. }
$$

In low-carbon and austenitic steels, zone 2 is in a plastic state. Let's write down the plasticity condition [5]

$$
\sigma_{r 2}^{2}+\sigma_{t 2}^{2}-\sigma_{r 2} \sigma_{t 2}-\sigma_{T}^{2}=0
$$

Taking into account (4), we write the differential equilibrium equation for our case in the for

$$
r \frac{d \sigma_{r 2}}{d r}=-\frac{\sigma_{r 2}}{2}-\sqrt{\sigma_{T}^{2}-\frac{3}{4} \sigma_{r 2}^{2}} .
$$


We write down the boundary conditions at the boundaries of the transition from one ring to another in the radial direction 1-2, 2-3

$$
r=r_{1} ; \sigma_{r 2}=\sigma_{r 1-2} ; \text { and } r=r_{2} ; \sigma_{r 2}=\sigma_{r 2-3}
$$

Taking into account (6), we find the solution of the differential equation (5) in the form

$$
\ln \frac{r_{2}}{r_{1}}=-\frac{1}{2} \ln \left(\frac{\sigma_{r 2-3}-2 \sqrt{\sigma_{T}^{2}-\frac{3}{4} \sigma_{r 2-3}^{2}}}{\sigma_{r 1-2}-2 \sqrt{\sigma_{T}^{2}-\frac{3}{4} \sigma_{r 1-2}^{2}}}\right)-\sqrt{\frac{3}{4}}\left[\arcsin \left(\sqrt{\frac{3}{4}} \frac{\sigma_{r 2-3}}{\sigma_{T}}\right)-\arcsin \left(\sqrt{\frac{3}{4}} \frac{\sigma_{r 1-2}}{\sigma_{T}}\right)\right] .
$$

It follows that a specific value of $\sigma_{r 1-2}$ in the radial direction corresponds to a specific value of the voltage $\sigma_{r 2-3}$ and a very specific distribution of stresses in zone 2 . The temperature stresses in the radial direction are determined by the formula [9]

$$
\sigma_{r}(t)=\frac{\alpha E \Delta T(t, r)}{2(1-v)}
$$

where $\Delta T(t, r)$, is the rate of temperature change in the radial direction.

To estimate the temperature stresses in the welded circuit, we will take into account the distribution of the temperature field in the contour of the welded joint, using the proposed graphoanalytic method, Fig. 2 (a, b, c). The temperature stresses obtained by calculation, which exceed the value of the yield strength, can be considered residual stresses in the weld contour.

\section{References}

[1] Mel'nikov, Design forms and methods of calculation of nuclear reactors, Atomizdat, Moskow, 1972.

[2] L.I. Mironova and I.I.Fedik, The local thermal loading of two intersecting cylindrical rotational shells with a variable wall thickness, J. of Machinery Manufacture and Reliability 44 (8) (2015) 720-725. https://doi.org/10.3103/S1052618815080026

[3] H.S. Carslaw and J.C. Jaeger, Conduction of Heat in Solids, Oxford, 1959.

[4] N. Rykalin, Calculation of thermal processes during welding, GLTIMN, Moskow, 1951.

[5] V.A. Vinokurov, Welding strains and stresses, Mashinostroenie, Moscow, 1968.

[6] R. Nigay, E. Nigay, L. Mironova, Investigation of thermal and deformation processes in the welding of shell structures made of carbon and high-alloy structural steels. J. of Physics: Conference Series, 1, (2020) 1431. https://doi.org/10.1088/1742-6596/1431/1/012039

[7] L. Mironova, R. Nigay, E. Nigay, About one method of modeling high-gradient temperature fields in the welding of shell structures made of carbon and high-alloy structural steels, IOP Conference Series: Materials Science and Engineering, 971(3) (2020) 032064. https://doi.org/10.1088/1757-899X/971/3/032064

[8] L. Mironova, R. Nigay, E. Nigay. Experimental and Computational Method for Determining Temperature Stresses in the Welding of Structures Made of Carbon and High-Alloy Structural 
Steels. J. Materials Science $\quad$ Forum, $1037 \quad$ (2021) 343-348. https://doi.org/10.4028/www.scientific.net/MSF.1037.343

[9] S. Timoshenko, Theory jf plates and shells, McGraw-Hill, New York, 1959.

[10] G.N. CHernyshev, A.L. Popov, V.M. Kozincev, I.I. Ponomarev, Residual stresses in deformable solids, Nauka, Fizmalit, Moscow, 1996.

[11]K. Gatovskij, V. Karhin, Theory of welding strains and stresses, LKI, Leningrad, 1980.

[12] G. Nikolaev, S. Kurkin, V. Vinokurov, Welded construction. Strength of welded joints and deformation of structures, Vysshaya shkola, Moscow, 1982. 